\title{
Localized Activation of p21-Activated Kinase Controls Neuronal Polarity and Morphology
}

\author{
Tom Jacobs, ${ }^{1}$ Frédéric Causeret, ${ }^{1}$ Yoshiaki V. Nishimura, ${ }^{2}$ Mami Terao, ${ }^{2}$ Adele Norman, ${ }^{1}$ Mikio Hoshino,${ }^{2}$ and \\ Margareta Nikolić ${ }^{1}$ \\ ${ }^{1}$ Department of Cellular and Molecular Neuroscience, Division of Neuroscience and Mental Health, Imperial College School of Medicine, Charing Cross \\ Campus, London W6 8RP, United Kingdom, and 2Department of Pathology and Tumor Biology, Kyoto University Graduate School of Medicine, Sakyo-ku, \\ Kyoto 606-8501, Japan
}

In the developing forebrain, neuronal polarization is a stepwise and initially reversible process that underlies correct migration and axon specification. Many aspects of cytoskeletal changes that accompany polarization are currently molecularly undefined and thus poorly understood. Here we reveal that the p21-activated kinase (Pak1) is essential for the specification of an axon and dendrites. In hippocampal neurons, activation of Pak1 is spatially restricted to the immature axon despite its uniform presence in all neurites. Hyperactivation of Pak1 at the membrane of all neurites or loss of Pak1 expression disrupts both neuronal morphology and the distinction between an axon and dendrites. We reveal that Pak1 acts on polarity in a kinase-dependent manner, by affecting the F-actin and microtubule cytoskeleton at least in part through Racl and cofilin. Our data are the first to demonstrate the importance of localized Pak1 kinase activation for neuronal polarization and differentiation.

Key words: Pak1 kinase; polarity; neuron; cytoskeleton; F-actin; axon

\section{Introduction}

Polarization of neurons is essential for their correct migration and subsequent specification of axons and dendrites. In the neocortex, hippocampus, and cerebellum, establishment of polarity correlates with the positioning of the centrosome and Golgi to the base of the leading process of a migrating neuron. An axon and dendrites are commonly specified by the location of the leading and trailing processes, suggesting that neuronal polarity is set at the time of migration (Noctor et al., 2004; Tsai and Gleeson, 2005; Solecki et al., 2006).

The molecular pathways of axonal specification are predominantly analyzed in vitro, where differentiation of hippocampal neurons is morphologically classified. Stage 2 neurons extend several uniform neurites. During stage 3, only one neurite becomes an axon, which matures together with dendrites in stages 4 and 5 (Dotti et al., 1988). The microtubule plus-end motor protein kinesin-1 transiently localizes to neurite terminals in a nonpolarized neuron but defines the emerging axon by stably accumulating in its tip (Jacobson et al., 2006). Phosphatidylinositol 3-kinase (PI3K) activation, subsequent enhancement of Akt, and

Received Feb. 20, 2007; revised May 31, 2007; accepted June 18, 2007.

This work was supported by a Wellcome Trust grant (M.N.). F.C. is a recipient of a long-term EMBO fellowship. We thank John Parnavelas and Sonja Rakić for the generous use of confocal microscopes; Phillip Gordon-Weeks for kindly providing anti-Map1b superBUGS antibody; Gary Bokoch and Jonathan Chernoff for pCMV6M-Pak1T423E; Ed Manser for phospho-Pak1 antibodies; Dan Wu for pSuper-Pak1 shRNA; David Turner for the mU6pro vector; Takeshi Kawauchi for technical advice; and Junichi Miyazaki and Takeshi Kawauchi for pCAG-IRES-EGFP.

Correspondence should be addressed to Dr. Margareta Nikolić, Department of Cellular and Molecular Neuroscience, Division of Neuroscience and Mental Health, Imperial College School of Medicine, Charing Cross Campus, Fulham Palace Road, London W6 8RP, UK. E-mail: m.nikolic@imperial.ac.uk.

DOI:10.1523/JNEUROSCI.0765-07.2007

Copyright $\odot 2007$ Society for Neuroscience $\quad$ 0270-6474/07/278604-12\$15.00/0 suppression of glycogen synthase kinase- $3 \beta$ (GSK3 $\beta$ ) kinases are required for axonal establishment and rapid outgrowth, whereas GSK $3 \beta$ remains active in the immature dendrites (Jiang et al., 2005; Wiggin et al., 2005). Cell surface proteins DOCK7, IGF receptor, and PMGS regulate the polarized activities of the Rho GTPases, Cdc42, Rac1, and RhoA (Da Silva et al., 2005; Sosa et al., 2006; Watabe-Uchida et al., 2006). Cdc42 controls the activity of JNK kinases and a polarity complex consisting of Par3, Par6, and atypical protein kinase C (Wiggin et al., 2005; Oliva et al., 2006). Inhibition of RhoA reduces recruitment of ROCK kinase and profilin IIa to the tip of the future axon, promoting its formation by destabilizing F-actin (Da Silva et al., 2005). Activation of Rac1 by the exchange factor TIAM1 or DOCK7 promotes axon formation in part by changing the activity of the microtubule-severing protein stathmin/Op18 (Watabe-Uchida et al., 2006). Neuronal polarization retains a degree of flexibility after its establishment because of the precise function of key proteins such as CRMP2, which controls axonal elongation by regulating microtubule assembly, reorganization of F-actin filaments, and endocytosis. CRMP2 loss of function causes the elaboration only of dendrites (Inagaki et al., 2001; Arimura and Kaibuchi, 2005, 2007).

The p21-activated kinase (Pak1) is a Cdc42 and Rac1 effector and a member of a family of six serine/threonine kinases classed into groups I (Pak1-Pak3) and II (Pak4-Pak6) (Jaffer and Chernoff, 2002; Bokoch, 2003). Pak1 activation requires GTPasedependent or -independent conformational changes leading to multiple phosphorylations that commonly occur at the plasma membrane. In Saccharomyces cerevisiae, Cryptococcus neoformans, and Dictyostelium, Pak kinases are essential for polarization (Ottilie et al., 1995; Holly and Blumer, 1999; Lee et al., 2004; Nichols et al., 2004). Mammalian Pak1 can facilitate directed 


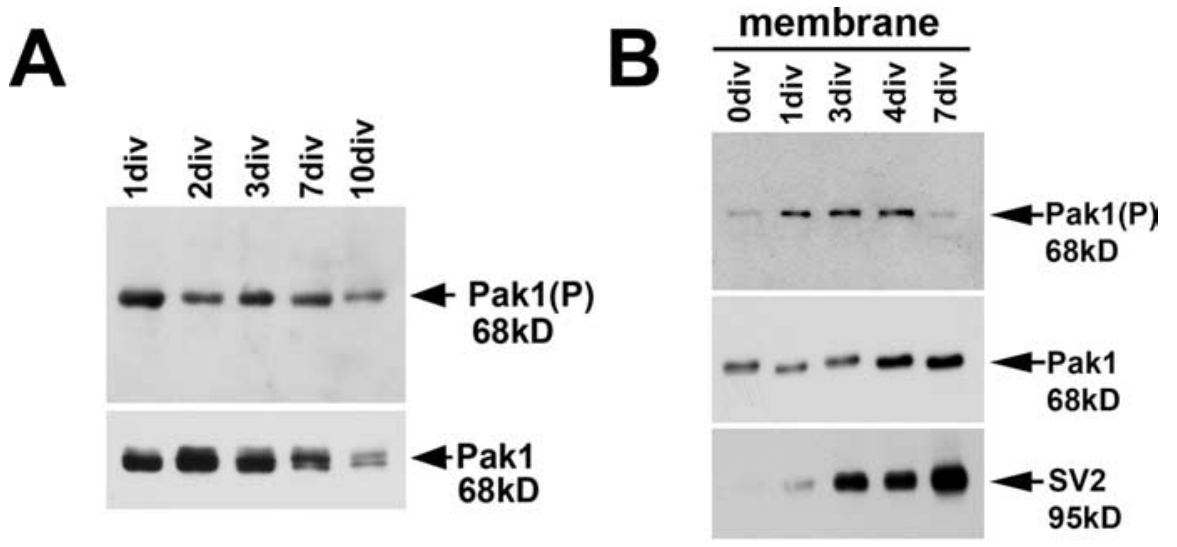

C

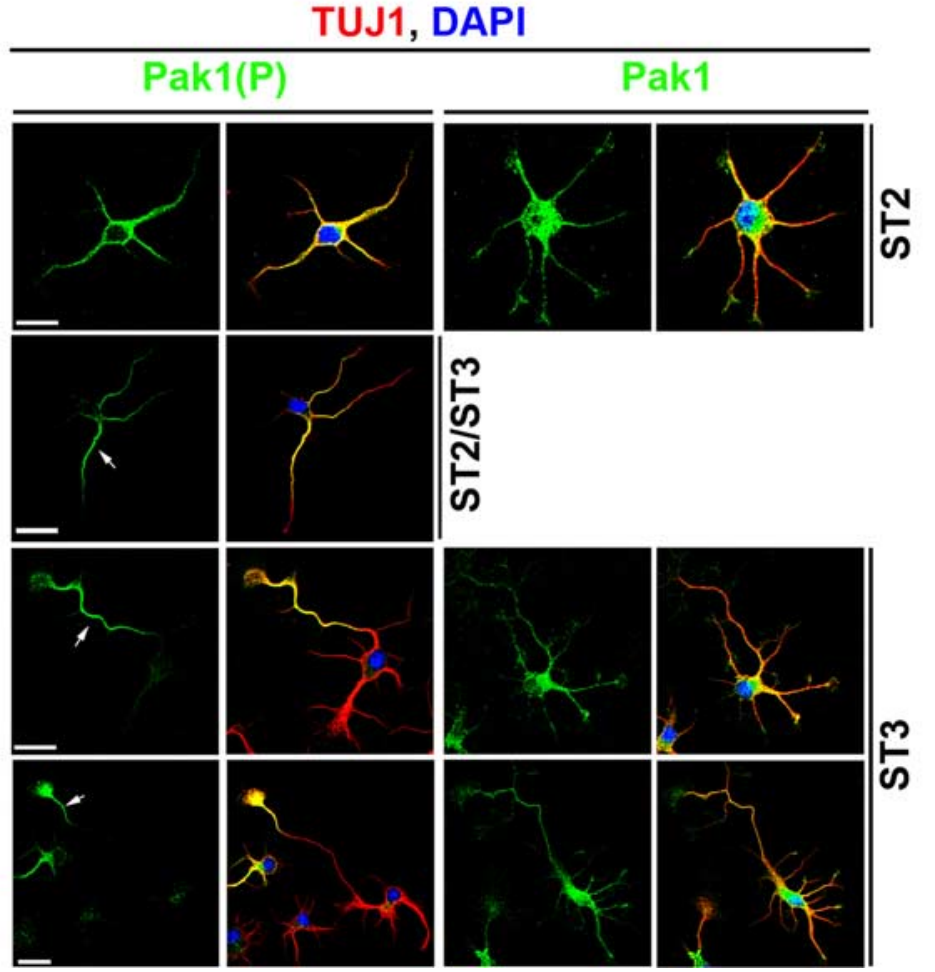

Figure 1. Polarized activation of Pak1. $A, B$, Cortical neurons were cultured for increasing lengths of time and examined for total and phosphorylated Pak1 by Western blot. $A$, The levels of Pak1(P) were highest during early stages of neuronal differentiation, paralleling the timing of axon specification and enhanced outgrowth. $\boldsymbol{B}, \operatorname{Pak} 1(\mathrm{P})$ was most evident in membranes between 1 and $4 \mathrm{~d}$ in vitro. Membrane enrichment was confirmed with SV2. C, Pak1 was uniformly distributed in all neurites of stage 2 (ST2) and 3 (ST3) hippocampal neurons. Pak1(P) showed overlapping distribution with total Pak1 in stage 2; however, at stage 3 it was exclusive to the longest neurite. A proportion of stage 2 neurons $(16 \%)$ increased Pak1(P) in one neurite, suggesting that they were at the boundary between stages 2 and 3 . The identity of all neurons was confirmed using the neuronal specific marker $\beta$-III tubulin (TUJ1), whereas 4',6' -diamidino-2-phenylindole (DAPI) staining revealed the nuclei. Scale bars, $25 \mu \mathrm{m}$.

migration of cultured fibroblasts and neutrophils (Dharmawardhane et al., 1999; Sells et al., 1999; Cau and Hall, 2005). We now reveal that localized activation of Pak1 is pivotal for neuronal polarity by affecting the function of proteins that regulate the F-actin and microtubule cytoskeleton.

\section{Materials and Methods}

Expression vectors. Catalytically active Pak1 (Pak1Caax) and inactive mutant (Pak1R299Caax) were subcloned into pCAG-IRES-EGFP vector using $\mathrm{ClaI}$ and BamHI. Enhanced green fluorescent protein (EGFP), GSTRacN17, GST-Cdc42N17, myc-Pak1T423E, pSuper Pak1 short hairpin RNA (shRNA), and cofilin and its constitutively active mutant
(cofilinS3A) were described previously (Nikolic et al., 1998; Kawauchi et al., 2003, 2006).

To generate shRNA expression vectors, an oligonucleotide targeting the coding region of Pak1 (sh1412, 5'-TTTGAGCCTTGTACCTCATTGCTTCAAGAGAGCAATGAGGTACAAGGCTCTTTTT-3') or control sequence (control shRNA, 5' -TTTGATGGATCGATATAGTGAGTTCAAGAGACTCACTATATCGATCCATCTTTTT- $3^{\prime}$ ) and their complementary sequences were inserted into $B b s \mathrm{I}$ and $\mathrm{XbaI}$ restriction sites of the mU6pro vector. All DNA preparations were made using plasmid purifications kits from Qiagen (Valencia, CA).

Antibodies and chemical reagents. The following antibodies were used in this study. AntiPak1 (N-20) and anti-Pak2 (V-19) (Santa Cruz Biotechnology, Santa Cruz, CA); anti-phospho Ser199/204 Pak1/Pak2 and anti-Pak3 (Millipore, Billerica, MA); anti-cofilin (Cell Signaling Technology, Beverly, MA); anti-phospho Ser3 cofilin, anti-dephosphorylated Tau (Tau-1), and anti-actin (Millipore); anti- $\beta$ III-tubulin (TUJ1) (Babco, Richmond, CA); antimicrotubule-associated protein 2 (Map2) (AP20), anti-vinculin, and anti-acetylated tubulin (Sigma-Aldrich, St. Louis, MO); antityrosinated tubulin (YL1/2) (AbD Serotec, Raleigh, NC); anti-SV2 (Developmental Studies Hybridoma Bank, University of Iowa, Iowa City, IA); and anti-phospho Thr 1265 Map1b (superBUGS) (Trivedi et al., 2005), anti-GFP, anti-mouse Alexa 488 or 568, and anti-rabbit Alexa 488, 568, or 633 (Invitrogen, Paisley, UK).

Jasplakinolide and cytochalasin D (CD) (EMD Biosciences, San Diego, CA) were applied at $5 \mathrm{~nm}$ and $1 \mu \mathrm{m}$ concentrations, respectively. All other chemicals were obtained from Sigma-Aldrich and VWR International (West Chester, PA).

Cell culture and transfection. Dissociated cortical or hippocampal neurons from embryonic day 17 (E17) to E19 rat brains were prepared as described previously (Nikolic et al., 1998) and plated on poly-D-lysine- $(16 \mu \mathrm{g} / \mathrm{ml})$ and lami$\operatorname{nin}(5 \mu \mathrm{g} / \mathrm{ml}$; Sigma-Aldrich)-coated glass or tissue culture plates. Neurons were transfected using a rat neuron nucleofector kit (Amaxa Biosystems, Gaithersburg, MD) according to the manufacturer's protocol and cultured in Neurobasal media supplemented with factor B27, 2 mM L-glutamine, $0.06 \mathrm{mg} / \mathrm{ml}$ cysteine, $1 \mathrm{~mm}$ sodium pyruvate, penicillin, and streptomycin (Invitrogen) at $37^{\circ} \mathrm{C}$ and $5 \% \mathrm{CO}_{2}$.

Immunocytochemistry. Neurons were fixed in 4\% paraformaldehyde for $20 \mathrm{~min}$, permeabilized in $0.2 \%$ Triton X-100, blocked in $0.2 \%$ fish skin gelatin, and immunostained as described previously (Nikolic et al., 1998). Images were captured using a Leica (Bannockburn, IL) TCS SP/UV confocal microscope and processed using Leica software.

Cell lysis and Western blotting. Cells were lysed in $25 \mathrm{~mm}$ Tris, $\mathrm{pH} 7.5$, $150 \mathrm{~mm} \mathrm{NaCl}, 5$ mм EDTA, 1\% Triton X-100, and 10\% glycerol with Complete protease inhibitors (Roche, Indianapolis, IN), 1 mM PMSF, 10 $\mathrm{mm} \mathrm{NaF}$, and $1 \mathrm{~mm} \mathrm{Na}_{3} \mathrm{VO}_{4}$. Lysates were cleared by centrifugation at $16,000 \times g$ for $10 \mathrm{~min}$. Membrane preparations were made as described previously (Nikolic et al., 1998). All lysates were separated by SDS-PAGE, Western blotted onto PVDF membranes, and probed as described pre- 
viously (Nikolic et al., 1998) using horseradish peroxidase-conjugated secondary antibodies (Vector Laboratories, Burlingame, CA) followed by incubation with ECL (GE Healthcare, Piscataway, NJ). Densitometric analysis of scanned films was used to compare the intensities of individual protein bands.

Kinase assays. Cells were lysed in $25 \mathrm{~mm}$ Tris, pH 7.5, 150 mм NaCl, 5 mм EDTA, 1\% Triton $\mathrm{X}-100$, and $10 \%$ glycerol with Complete protease inhibitors (Roche), $1 \mathrm{~mm}$ PMSF, $10 \mathrm{~mm}$ $\mathrm{NaF}$, and $1 \mathrm{~mm} \mathrm{Na}_{3} \mathrm{VO}_{4}$. Lysates were cleared by centrifugation at $16,000 \times g$ for $10 \mathrm{~min}$. One hundred to two hundred micrograms of lysate were immunoprecipitated with appropriate primary antibody and protein A-Sepharose beads and subsequently washed multiple times in kinase buffer (50 mM HEPES, pH 7.5, and 10 $\mathrm{mm} \mathrm{MgCl}_{2}$ ) as described previously (Nikolic et al., 1998; Rashid et al., 2001). Kinase assays were performed in the presence of $50 \mu \mathrm{M} \mathrm{ATP}, 1 \mu \mathrm{Ci}$ $\left[{ }^{32} \mathrm{P} \gamma\right.$-ATP] (GE Healthcare), $2 \mu \mathrm{g}$ of histone H4 (Roche), 1 mм DTT, and kinase buffer for $20 \mathrm{~min}$ at $30^{\circ} \mathrm{C}$. The reactions were stopped with $2 \times$ sample buffer, resolved by SDS-PAGE, dried, and exposed to autoradiography.

Measurements. Neurons were examined using a Nikon (Tokyo, Japan) TE2000 microscope and Plan Fluor $20 \times / 0.45$ objective, images were captured with a Hamamatsu (Hamamatsu, Japan) Orca camera and Openlabs software (Improvision, Lexington, MA). Neurite lengths were measured using Volocity 4.0 software (Improvision) with any length $<10 \mu \mathrm{m}$ discounted. The effects of Pak1Caax and EGFP on neuronal morphology after 1-3 d in culture were scored from $>120$ neurons $(n=$ 3). Data for EGFP- and Pak1Caax-expressing neurons after $7 \mathrm{~d}$ in vitro (div) were pooled from all experiments $(n=14)$ and are therefore based on the scoring of $>1000$ neurons. For comparisons between outgrowth at 3 and $7 \mathrm{div}$, data were collected from $>200$ neurons $(n=2)$. The effects of Pak1R299Caax, Pak1T423E, and Cdc42N17 were analyzed from $>200$ neurons $(n=2)$, the consequences of cofilinS3A and RacN17 were analyzed in $>400$ neurons $(n=4)$, and the effects of jasplakinolide were examined in 300 neurons $(n=3)$. To determine the distribution of phosphorylation of Pak1 on S199/204 [Pak1(P)], images of $>100$ neurons $(n=3)$ were captured using constant-exposure settings and analyzed with Openlabs software. To measure the distribution of Map2, images of $>140$ neurons $(n=3)$ were captured using constant-exposure settings and analyzed with Openlabs software.

\section{Results}

\section{Polarized activation of Pak1}

Pak1 activation is accompanied by sequential phosphorylation catalyzed by Akt/PKB, PDK1, and Pak1 (Bokoch, 2003). Phosphorylation of S199/204 and T423 were shown to strongly correlate with an increase in Pak1 catalytic activity and are widely used as markers of its activation (King et al., 2000; Sells et al., 2000; Chong et al., 2001; Loo et al., 2004; Zhao et al., 2005). We examined the activation profile of Pak1 by following the levels of Pak1(P) during differentiation of cultured cortical neurons. Western blots revealed high levels of Pak1(P) from 1 to $7 \mathrm{~d}$ in culture with a subsequent decrease in both total and phosphorylated Pak1 (Fig. 1A). Because the phospho-specific antibody can also detect Pak2 and possibly Pak3, it is interesting to note that we only observed one band on our Western blots that corresponded erebral cortex
DAPI

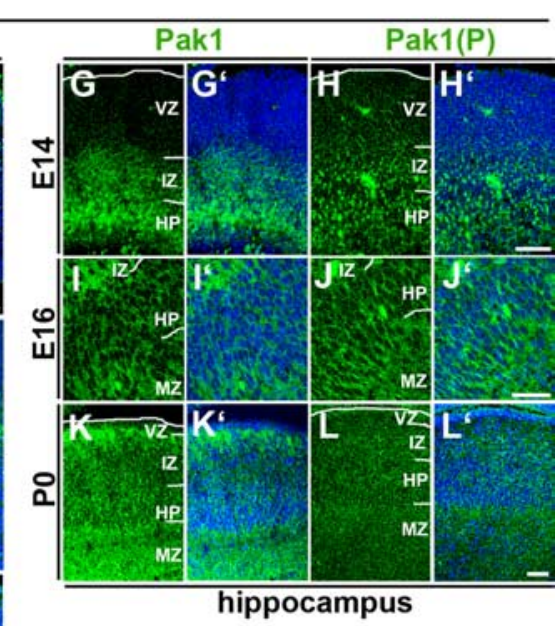

hippocampus

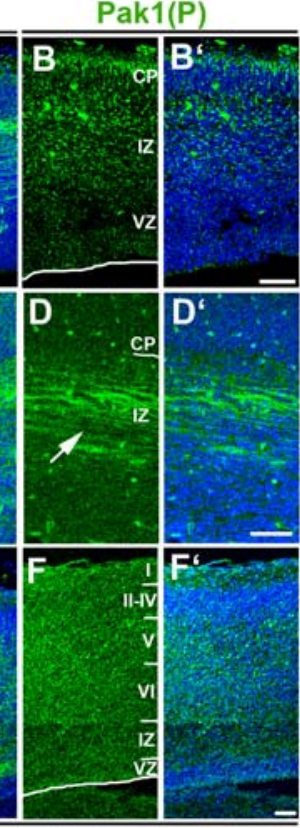

Figure 2. The developmental pattern of Pak1 expression and activation. $\boldsymbol{A}-\boldsymbol{L}$, Immunodetection of Pak1 $(\boldsymbol{A}, \boldsymbol{C}, \boldsymbol{E}, \boldsymbol{G}, \boldsymbol{I}, \boldsymbol{K})$ and Pak1 phosphorylated on $\mathbf{S 1 9 9 / 2 0 4}(\boldsymbol{B}, \boldsymbol{D}, \boldsymbol{F}, \boldsymbol{H}, \boldsymbol{J}, \boldsymbol{L})$ in the developing cortex $(\boldsymbol{A}-\boldsymbol{F})$ and hippocampus $(\boldsymbol{G}-\boldsymbol{L}) ; 4^{\prime}, \mathbf{6}^{\prime}$-diamidino-2in (DAPI; blue) revealed nuclei of all cells. $A, A^{\prime}$, InE14 mouse, cerebral cortex Pak1 is highly enriched in the axons of the resemble that seen at E14 with highest levels in the IZ, HP, and marginal zone (MZ). $K, \boldsymbol{L}$, Pak1 remains high at birth in the IZ, HP and $M Z$, whereas Pak1 $(P)$ is downregulated, with highest levels evident in the $M Z$ and a relatively uniform distribution in the IZ and HP. Scale bars: $\boldsymbol{B}^{\prime}$ (for $\boldsymbol{A}-\boldsymbol{B}^{\prime}$ ), $\boldsymbol{D}^{\prime}$ (for $\boldsymbol{C}-\boldsymbol{D}^{\prime}$ ), $\boldsymbol{F}^{\prime}$ (for $\boldsymbol{E}-\boldsymbol{F}^{\prime}$ ), $\boldsymbol{H}^{\prime}$ (for $\boldsymbol{G}-\boldsymbol{H}^{\prime}$ ), $\boldsymbol{L}^{\prime}$ (for $\boldsymbol{K}-\boldsymbol{L}^{\prime}$ ), $45 \mu \mathrm{m} ; \boldsymbol{I}, \boldsymbol{J}, 30 \mu \mathrm{m}$.

to Pak1, suggesting that in these cultures it is the predominant group I Pak kinase. We compared the subcellular distribution of total and S199/204 phosphorylated Pak1 in cultured cortical and hippocampal neurons. Western blots revealed a transient enrichment of Pak1(P) in membrane fractions derived from cortical cultures that peaked at 2-4 div and dramatically dropped at $7 \mathrm{div}$ (Fig. $1 B$ ). The timing of high membrane Pak1(P) coincided with the transition of most neurons from a nonpolarized to a polarized state (stages 2-3). In contrast, membrane levels of total Pak1 increased with time in culture, remaining high after 4 div. At stage 1, when hippocampal neurons have not yet elaborated neurites, Pak1 was asymmetrically enriched and activated in an area encompassing the Golgi (data not shown). In stage 2 neurons, which have extended multiple neurites but not yet polarized, Pak1 was uniformly distributed and activated. Interestingly, on average $16 \pm 1.5 \%$ of stage 2 neurons had higher levels of Pak $1(\mathrm{P})$ in a single neurite (Fig. 1C). By stage 3 , when neurons undergo specification of a future axon, active Pak1 was evident only in the cell soma and the distal half of the longest neurite, despite continued uniform distribution of total Pak1 (Fig. 1C). These results reveal that polarization of hippocampal and cortical neurons is accompanied by asymmetric activation of Pak1.

To determine whether Pak1 expression and phosphorylation accompany neuronal differentiation in vivo, we examined the developing cerebral cortex and hippocampus at mouse E14 and 
A
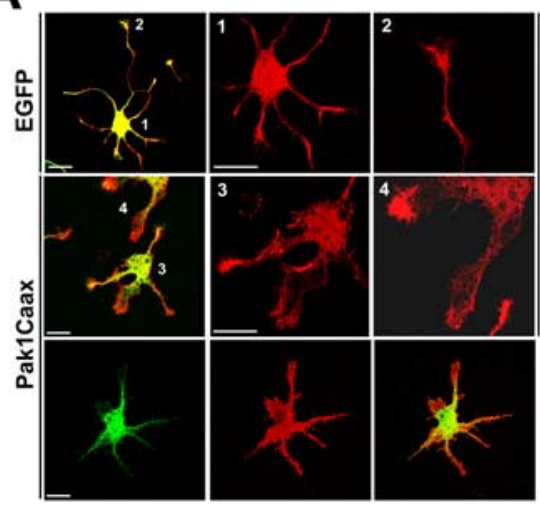

C

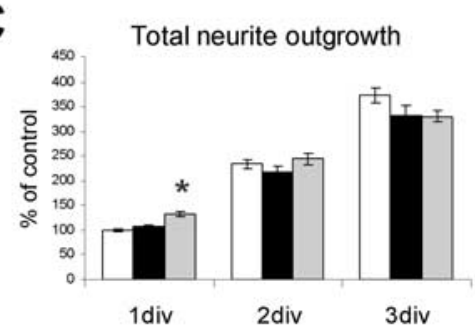

E

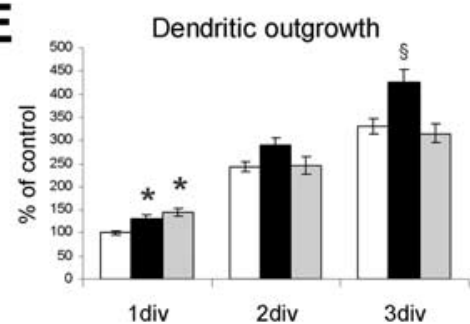

B

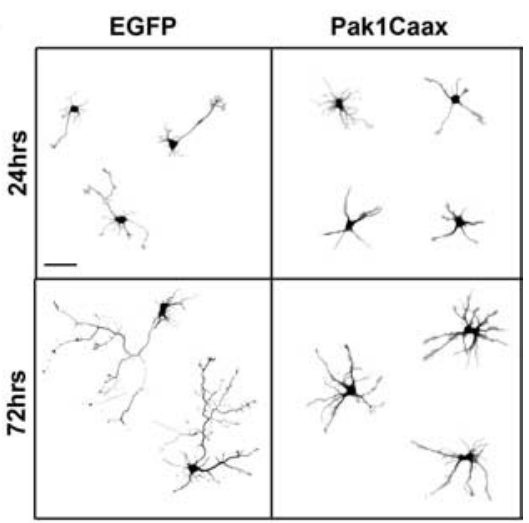

D

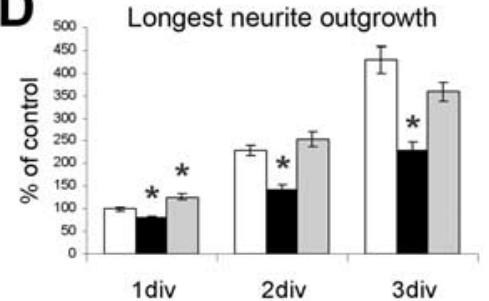

$\square$ GFP

- Pak1Caax

Pak1R299Caax

Figure 3. Overexpression of catalytically active Pak 1 alters neuronal morphology. A, Neurons expressing Pak1Caax after 3 div had more lamellipodia than the EGFP-expressing controls. Constitutive activation and membrane localization of Pak1Caax was confirmed using the anti-S199/204 Pak1(P) antibody. Scale bars, $25 \mu \mathrm{m}$. B, Morphological comparison of EGFP- and Pak1Caaxexpressing neurons. Note that Pak1Caax-positive neurons did not have a distinguishable longest neurite after 1 or 3 div. Scale bar, $50 \mu \mathrm{m}$. C, Total neurite outgrowth was normalized to the EGFP 1 div control. No significant differences were evident between expression of EGFP and Pak1Caax, whereas a 33\% increased outgrowth was evident in Pak1R299Caax-positive neurons only at 1 div. D, The longest neurite was measured revealing a significant reduction in length after Pak1Caax expression. Pak1R299Caax enhanced outgrowth by $25 \%$ only at 1 div. $E$, Remaining neurites were significantly longer in Pak1Caax neurons when compared with EGFP controls at 1 and 3 div. Pak1R229Caax enhanced outgrowth only at 1 div. Error bars depict SEM. ${ }^{\S} p<0.01$ and ${ }^{*} p<$ 0.001 using Student's $t$ test.

E16 and at birth [postnatal day 0 (P0)]. In confirmation with our previous findings, Pak1 levels were low in the proliferating ventricular zones (VZs) and high in the axon-rich intermediate zones (IZs) of the cerebral cortex and hippocampus in all examined ages (Fig. 2) (Zhong et al., 2003). Development of the cortex was marked by progressive enrichment of Pak1 in the cortical plate (CP) from E14 to P0 (Fig. 2A,C,E), whereas high levels were already present in the hippocampal plate (HP) at E14 (Fig. 2G). In embryonic stages, the distribution of phosphorylated Pak1 was similar but not as extensive as that of total Pak1. Small foci of concentrated Pak1 (P) were seen in the E14 cortex and hippocampus primarily in the IZ, CP, and HP (Fig. $2 B, H$ ). At E16, Pak1(P) accumulated more broadly in axonal tracts of the IZ and neurons of the CP and HP (Fig. 2D,J). Phosphorylated Pak1 remained high in the $\mathrm{CP}$ and HP but was reduced in the IZ at birth (Fig. $2 F, L)$. These results were consistent with the segregation of Pak1 $(\mathrm{P})$ to the distal portion of the axon in polarized hippocampal neurons and its subsequent downregulation observed in vitro
(Fig. 1C). At all stages, lowest levels of Pak1(P) were seen in the VZ.

Pak1 affects neuronal morphology

Pak1 exists in the cytoplasm in an inactive homodimerized state and can be activated by recruitment to the membrane (Lei et al., 2000; Bokoch, 2003). Because membrane enrichment of activated Pak1 coincides with the timing of axon specification, we examined the consequences of Pak1 hyperactivation using a protein that has a Ras prenylation sequence (Caax box) at its C-terminal domain (Pak1Caax), which renders it constitutively active (Daniels et al., 1998). This also provided us with an ideal tool to disrupt the asymmetry of endogenously activated Pak1. To verify that in neurons fusion of Pak1 to the Caax box results in its activation at the membrane, we compared the levels of phosphorylated Pak1 in membrane fractions derived from Pak1Caax- and EGFP-expressing cortical neurons. Clear activation of Pak1Caax was evident, confirming previous reports (supplemental Fig. $1 \mathrm{~A}$, available at www. jneurosci.org as supplemental material) (Daniels et al., 1998). We also examined the levels and activities of Pak2 and Pak3, observing no detectable changes after Pak1Caax expression (supplemental Fig. $1 B$, available at www.jneurosci.org as supplemental material). Hippocampal neurons were transfected with Pak1Caax or its inactive mutant, Pak1R299Caax, and examined after 1,2, or $3 \mathrm{~d}$. Neurons expressing Pak1Caax displayed increased lamellipodia and a star-like morphology with all neurites of apparently similar lengths, a phenotype not observed after Pak1R299Caax or EGFP expression (Fig. $3 A, B)$. Overexpression of Pak1Caax reduced outgrowth of the longest neurite (which was considered the most likely future axon), whereas the remaining neurites (considered future dendrites) extended farther than the EGFP controls (Fig. 3D,E). Expression of the Pak1R299Caax mutant had transient and minor consequences on neurite outgrowth and was otherwise indistinguishable from EGFP controls (Fig. 3C-E). Our data suggest that in hippocampal neurons, localized activation of Pak1 in a single neurite is necessary for correct axon and dendrite morphology.

\section{Activation of Pak1 disrupts specification of axons and dendrites}

In mature, polarized hippocampal neurons, Pak1(P) was only present in axons, where it cosegregated with the axonal marker Tau-1 (Fig. 4A). In contrast, total Pak1 remained high in both dendrites and axons (Fig. 4A). Because activation of Pak1 at the membrane differentially affected neurite outgrowth and morphology, we examined the consequences of its expression on the specification of axons and dendrites. Transfected neurons were cultured for 3 or $7 \mathrm{~d}$ before fixation when axons and dendrites 
were identified by expression of Tau- 1 and Map2, respectively. At 3 div, hyperactivation of Pak1 caused on average $35.2 \pm$ $0.6 \%$ of the neurons to elaborate more than one Tau-1-positive neurite, in contrast to $17.4 \pm 0.3 \%$ of EGFP controls (supplemental Fig. 2, available at www. jneurosci.org as supplemental material). This phenotype became increasingly apparent with time in culture; thus, at 7 div, $60.8 \pm 1.5 \%$ of Pak1Caax-expressing neurons had more than one Tau-1-positive neurite, in contrast to an average of $21.7 \pm$ $1.8 \%$ of EGFP controls and $28.2 \pm 0.2 \%$ of catalytically inactive Pak1R299Caax (Fig. $4 B, D)$. To investigate whether the effects of Pak1 on polarization depended on its membrane localization, we overexpressed a constitutively active Pak1T423E mutant. Neurons expressing Pak1T423E exhibited increased lamellipodia but normal polarity with only $16.6 \pm 2 \%$ elaborating multiple Tau-1-positive neurites, confirming that Pak1 affects polarity when at the membrane (Fig. 4C,D).

In EGFP-expressing controls, the segregation of Map2 to dendrites and absence from axons was evident at 3 div and highly apparent at 7 div. In contrast, in many Pak1Caax-expressing neurons Map2 extended farther down all neurites at 3 and 7 div (Fig. 5A). We measured the area of a cell that contained Map2, revealing that after 3 div on average $59.6 \pm 6.9 \%$ of a Pak1Caax-expressing neuron had Map2 in contrast to $38.7 \pm 6.7 \%$ of an EGFPexpressing control. The differences remained apparent at 7 div when on average $25.6 \pm 0.2 \%$ of a control cell had Map2, in contrast to $40.8 \pm 2.8 \%$ of a Pak1Caaxexpressing neuron (Fig. 5B). Because of the extended presence of Map2 in axon-like neurites, many of which were Tau-1 positive, we used a later axonal marker, Map1b phosphorylated on T1265 [Map1b(P)], a known GSK3 $\beta$ target site (Trivedi et al., 2005). In $81.9 \pm 0.9 \%$ of EGFP-expressing controls, this marker only labeled a single axon (Fig. $5 C$ ). In $24.2 \pm 4.9 \%$ of Pak1Caax-expressing neurons, Map 1b(P) labeled multiple long neurites, suggesting the formation of several axonlike neurites (Fig. 5D). Unexpectedly, in $32.9 \pm 3.8 \%$ of Pak1Caax-expressing neurons, Map1b(P) was not detectable in any neurites. These neurons always had stage 2-like morphologies, suggesting that they had not transferred to stage 3 . Together, our results suggest that many neurons respond to Pak1Caax overexpression by failing to specify an axon and dendrites. Pak1Caax can also cause neurons to elaborate multiple Tau-1-, Map1b(P)-positive axon-like neurites. This variation of consequences may reflect the levels and timing of Pak1Caax expression in relation to the differentiation status of the targeted neuron.

\section{Pak1 is required for neurite specification and neuronal viability}

To examine the role of endogenous Pak1, we used two shRNA expression vectors, one of which was previously used to demon-
Pak1(P), DAPI
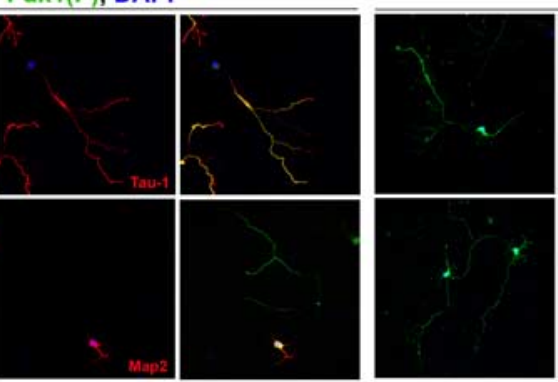

Pak1, DAPI

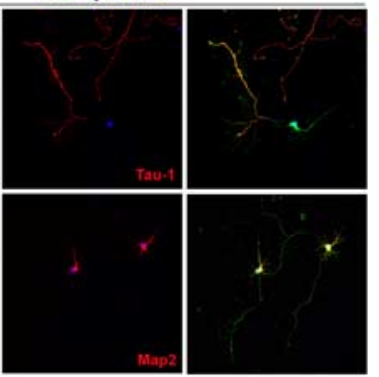

Map2, Tau-1

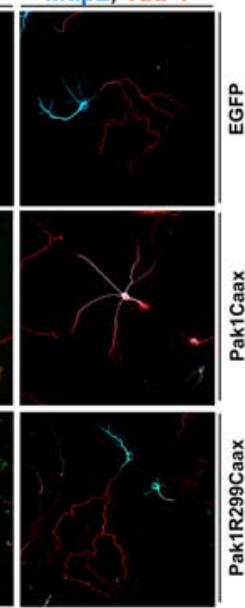

Pak1, Map2
C

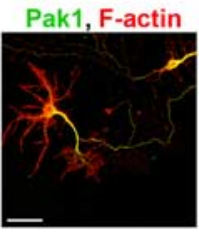
Pak1, Tau-1
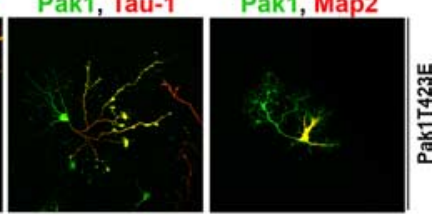

D

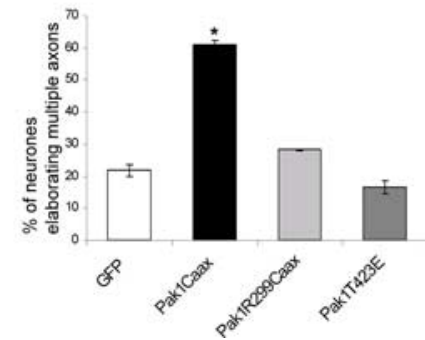

Figure 4. Membrane localization of catalytically active Pak1 induces multiple axons. $\boldsymbol{A}$, The subcellular distribution of $\mathrm{S199}$ / (Tau-1) and dendrites (Map2). Pak1(P) was detected only in axons despite uniform presence of Pak1 in axons and dendrites. Nuclei wisualized by $4^{\prime}, 6^{\prime}$-diamidino-2-phenylindole (DAPI) staining. B, C, After 7 div, expression of Pak1Caax alters the distribuxon and 1 and Map2. In contrast, neurons expressing Pak1R299, EGFP, or Pak1T423E had segregated Tau- 1 and Map2 to an expressing neurons elaborated multiple axons in $60.8 \pm 1.5 \%$ of cases in contrast to Pak1R299Caax ( $28.2 \pm 0.2 \%)$, EGFP( $21.7 \pm$ $1.8 \%)$, and Pak1T423E (16.6 $\pm 2 \%)$. Scale bars, $50 \mu \mathrm{m}$. Error bars represent SEM. ${ }^{*} p<0.001$ using Student's $t$ test.

strate Pak1 importance for neutrophil chemotaxis ( $\mathrm{Li}$ et al., 2003). The effectiveness of the shRNA vectors was verified by transfection into Pak1-expressing Cos7 cells or cortical neurons, which were analyzed by Western blotting (Fig. 6A,B). We also verified that downregulation of Pak1 activity had no significant effect on the expression levels and activities of Pak2 or Pak3 (supplemental Fig. $1 B$, available at www.jneurosci.org as supplemental material). In hippocampal neurons, expression of either Pak1 shRNA caused the appearance of aberrant F-actin-rich areas of cell spreading around the soma and along the neurites (Fig. 6C). The somal protrusions in particular contained an abundance of curved microtubules, many of which were acetylated and thus stabilized (Fig. 6D). The formation of extensive lamellipodia was not observed in EGFP or control shRNA-expressing neurons. After 3 div, $38 \pm 3.6 \%$ of Pak1 shRNA-expressing neurons had disorganized neurites that were both Tau-1 and Map2 positive, in contrast to the control shRNA neurons, of which only $15.3 \pm$ $3.3 \%$ had not polarized ( ${ }^{*} p<0.01$ using Student's $t$ test) (Fig. 7). At 3 div, Pak1 shRNA expression caused a 13\% reduction in total neurite outgrowth when compared with control shRNA. Unexpectedly, at 7 div, expression of Pak1 shRNA caused neuronal lethality (supplemental Fig. 3, available at www.jneurosci.org as 
A
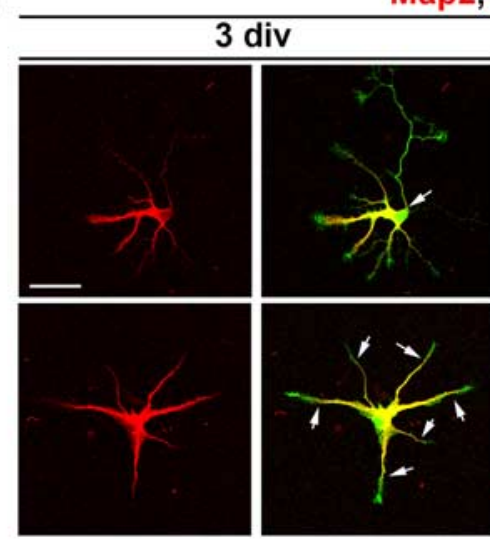

B

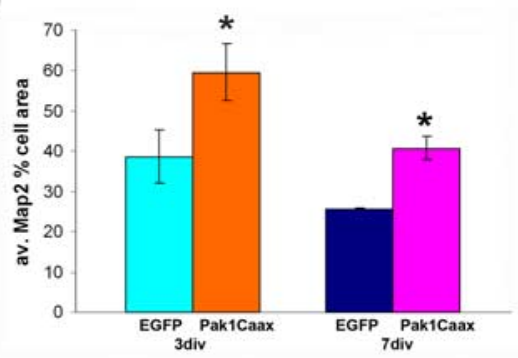

D
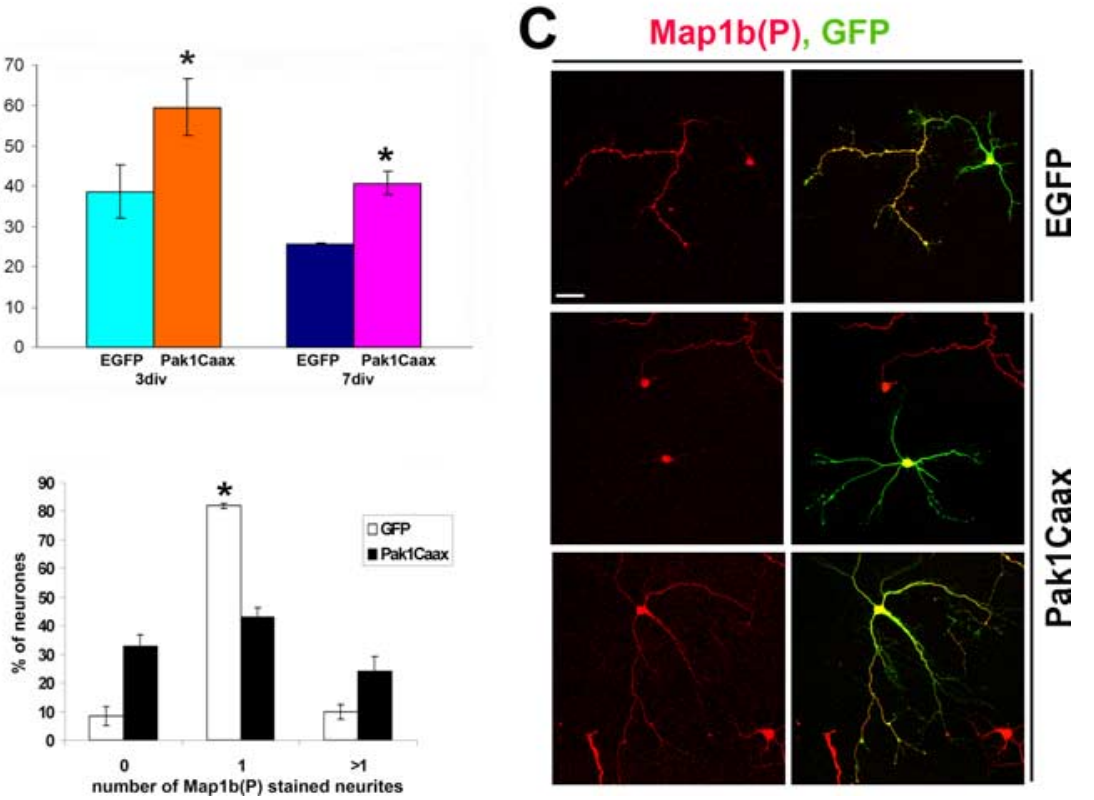

Figure 5. Pak1 hyperactivation affects the distribution of microtubule-associated proteins. A, Pak1Caax-expressing neurons have a broader distribution of Map2 at 3 and 7 div than EGFP controls. Arrows mark the extent of Map2 presence in individual neurites. $\boldsymbol{B}$, The average (av.) percentage of a cell area that contains Map2 is greater after Pak1Caax expression than in EGFP controls. C, Two representative examples show Pak1Caax-induced absence of Map1b(P) or widespread distribution of Map 1b(P) in all neurites. $D$, Neurons were scored for the absence of $M a p 1 b(P)$ or its presence in one or multiple neurites. Scale bars, $50 \mu \mathrm{m}$. Error bars represent SEM. ${ }^{*} p<0.001$ using Student's $t$ test.

supplemental material). Comparing the numbers of live targeted cells at 3 and 7 div revealed a $8.3 \pm 3.3 \%$ viability after Pak1 shRNA expression, in contrast to $80.6 \pm 6.1 \%$ seen in the shRNA control. Some neurons that remained viable at $7 \mathrm{div}$ had no visible neurites, whereas others had extended one or a few highly disorganized processes. Lamellipodia rich in F-actin and microtubules remained evident particularly in large somal extensions (supplemental Fig. 3, available at www. jneurosci.org as supplemental material). The loss of neuronal viability coupled with the severely altered morphologies prevented us from scoring the number of polarized neurons.

\section{Polarization depends on Pak1 and F-actin}

Neuronal polarization is characterized by local destabilization of F-actin in the growth cone of one neurite, which will subsequently become the axon. Local application of low doses of the F-actin-destabilizing drug $\mathrm{CD}$ can induce a neurite to become an axon (Bradke and Dotti, 1999, 2000). To examine Pakl activation in response to the induction of multiple axons, we exposed cultured hippocampal neurons to low doses $(1 \mu \mathrm{M})$ of $\mathrm{CD}$ a few hours after plating, when all neurons had stably attached to the substratum. After $7 \mathrm{~d}$ in culture, $\mathrm{CD}$ treatment resulted in the appearance of hippocampal neurons elaborating multiple axons that were positive for Tau- 1 and Map1b(P) (Fig. $8 A$ ) (data not shown). Interestingly, all of the Tau-1-positive neurites also had high levels of Pak1(P), suggesting that Pak1 activation is responsive to changes in the F-actin cytoskeleton and accompanies axon formation.

Because CD can cause Pak1 activation, we attempted to rescue the effects of Pak1Caax expression by exposure to low doses of the F-actin-stabilizing drug jasplakinolide. Hippocampal neurons transfected with EGFP alone or with Pak1Caax were treated a few hours after plating with $5 \mathrm{~nm}$ jasplakinolide and maintained in culture for $7 \mathrm{~d}$. Long-term exposure to jasplakinolide caused an increase in disorganized lamellipodial protrusions of both EGFP- and Pak1Caax-expressing neurons (Fig. 8 B). Jasplakinolide partially rescued the Pak1Caax phenotype, reducing the percentage of neurons with multiple Tau1 -positive neurites from $60.8 \pm 1.5 \%$ to $41.6 \pm 1.4 \%$ (Fig. $8 C$ ). No significant effect on polarity was observed in the EGFP controls. Together, these data suggest that a dynamic interplay exists between F-actin organization and Pak1 activity, which is required for axon formation in hippocampal neurons.

\section{Pak1 regulates neuronal polarity through activation of Rac1 and inhibition of cofilin}

It is well established that both $\mathrm{Cdc} 42$ and Racl can activate the group I Pak kinases (Bokoch, 2003). It has also transpired that Pak1 can induce Racl activation through the association with PIX/Cool exchange factors or inhibitory phosphorylation of the Rac dissociation inhibitor RhoGDI (Manser et al., 1998; Obermeier et al., 1998; DerMardirossian et al., 2004). Importantly, association of Pak1 and the exchange factors PIX/Cool was recently shown to be responsible for the formation of polarized lamellipodia in epithelial cells (Cau and Hall, 2005). To investigate how Pak1 regulates neuronal morphology and polarity, we attempted to rescue the Pak1Caax phenotype by coexpression with a dominant-negative mutant of Racl, Rac1N17. At 7 div, coexpression of RacN17 reduced the percentage of neurons extending multiple axons from $60.8 \pm 1.5 \%$ (Pak1Caax) to $33.4 \pm$ 5.3\% (Pak1Caax plus RacN17) (Fig. 9A,C). However, RacN17 did not rescue the effects of Pak1Caax on neuronal morphology and lamellipodia extension. Expression of RacN17 alone decreased the number of neurons elaborating multiple axons from $21.7 \pm 1.8 \%$ in the EGFP control group to $13 \pm 0.5 \%$ 
(supplemental Fig. 4, available at www. jneurosci.org as supplemental material). In contrast, inhibition of Cdc42 by coexpression of the Cdc42N17 mutant with Pak1Caax had no significant effect on the number of Tau-1- and Map2-positive neurites (Fig. 9A; supplemental Fig. 4, available at www.jneurosci.org as supplemental material). To further examine whether Racl acts upstream and downstream from Pak1Caax, we compared the levels of Pak1Caax activation in cortical neurons in the presence or absence of coexpressed RacN17, revealing no significant differences (supplemental Fig. 1A, available at www.jneurosci.org as supplemental material). These data suggest that Rac1 lies downstream from the polarizing effects of Pak1.

Pak1 is known to affect F-actin organization by phosphorylating and activating the LIM-1 kinase (LIMK-1) (Edwards et al., 1999). This results in an increase in LIMK-1 activity toward a family of F-actin-depolymerizing and -severing proteins, cofilin/ADF. Phosphorylation of cofilin on S3 is inhibitory, reducing its binding affinity toward F-actin (Sarmiere and Bamburg, 2004). To determine whether Pak1Caax overexpression in forebrain neurons results in cofilin inactivation, we examined the levels of S3 phosphorylated cofilin, revealing an approximately threefold increase in lysates obtained from Pak1Caax-transfected neurons when compared with the EGFPexpressing controls (Fig. 9B). This is most likely underestimated, because the transfection efficiency ranged between 40 and $60 \%$. To determine whether cofilin phosphorylation is required for the effects of Pak1 on neuronal polarity and morphology, we coexpressed a nonphosphorylatable, constitutively active mutant (cofilinS3A) with Pak1Caax in hippocampal neurons. Constitutively active cofilin did not reduce the effects of Pak1Caax on axonal outgrowth and branching (data not shown). However, the percentage of neurons with multiple, Tau-1 immunoreactive axons decreased from $60.8 \pm 1.5 \%$ (Pak1Caax) to $39.3 \pm$ 2.7\% (Pak1Caax plus cofilinS3A) (Fig. 9A,C). These data further confirm that Pak1 acts on neuronal polarity by affecting F-actin organization and identify cofilin as its likely target.

\section{Discussion}

Postnatal inhibition of Pak1-Pak3 causes a morphological change and reduced number of dendritic spines, which in mice results in impaired memory consolidation (Hayashi et al., 2004). In vitro Pak1 can act on spine morphogenesis by phosphorylating the myosin II regulatory light chain and as a downstream target of ephrinB1/EphB receptor signaling (Penzes et al., 2003; Zhang et al., 2005). Therefore, once the forebrain is formed, Pak1 has a

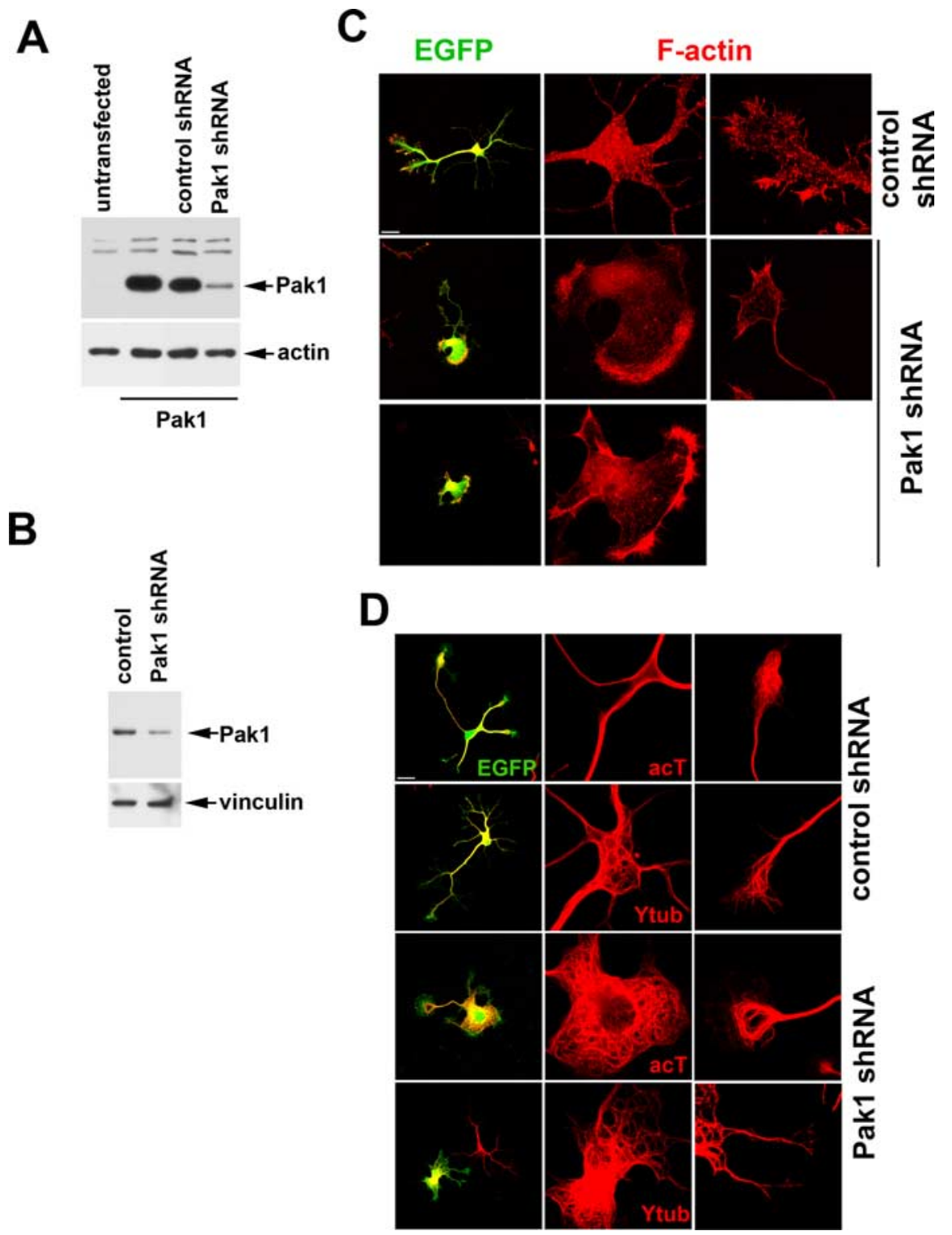

Figure 6. Loss of Pak1 expression affects the neuronal cytoskeleton. $A$, The levels of Pak1 in transfected C $\operatorname{Cos} 7$ cells were reduced after coexpression with Pak1 shRNA but remained unchanged in the presence of a control shRNA. $\boldsymbol{B}$, Cortical cultures transfected with empty vector (control) or Pak1 shRNA were examined by Western blot after 3 div, revealing a reduction in Pak1 protein levels. no neurites were evident. This phenotype was not observed after expression of control shRNA. D, At 3 div, expression of Pak1 ShRNA caused the presence of many looped microtubules, particularly in the somal lamellipodia. Many of the microtubules were stable as judged by the presence of acetylated tubulin (acT). An antibody to tyrosinated tubulin (Ytub) revealed the presence of newly formed microtubules in both control and Pak1 shRNA-expressing neurons. Scale bars, $50 \mu \mathrm{m}$.

major postsynaptic role; however, its importance for early stages of neuronal development has not been elucidated. Our study is the first to demonstrate a pivotal role for Pak1 for the specification and outgrowth of an axon and dendrites.

Hippocampal neurons provide a well established model for studying neuronal differentiation in vitro. We demonstrate that asymmetric activation of Pak1 occurs as a hippocampal neuron transitions from a nonpolarized to an axon specified stage. Enrichment of active Pak1 continues to predominate in the axon, suggesting that active Pak1 is subsequently required for the outgrowth and function of the axon. Interestingly, all of the multiple axons that formed as a consequence of exposure to CD had increased Pak1 $(\mathrm{P})$, confirming that Pak1 activation is an integral 
3 div

Tau-1, EGFP
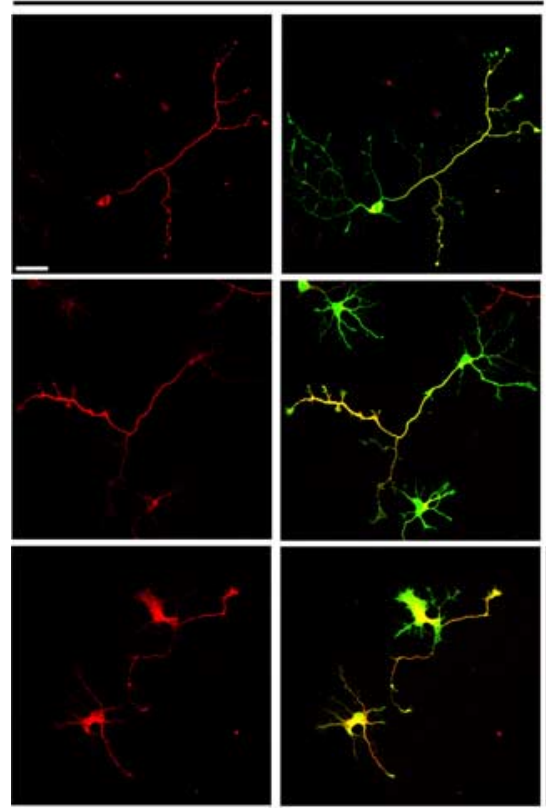

Figure 7. Inhibition of Pak1 affects neuronal polarization. After 3 div, neurons expressing Pak1 shRNA revealed a widespread distribution of Tau-1 and Map2 in contrast to EGFP or control shRNA-transfected neurons, in which the markers segregated to axons and dendrites, respectively. Scale bar, $50 \mu \mathrm{m}$.

aspect of axon formation and growth. The fact that F-actin destabilization caused Pak1 activation also suggests the existence of feedback mechanisms from actin to its regulatory proteins, which ultimately control the balance between its polymerization and depolymerization in a temporally and spatially regulated manner.

The consequences of Pak1 loss of function on neuronal differentiation are striking. If Pak1 was only required for axonal specification, we would have predicted that its downregulation would induce the formation only of dendrites, in a similar manner to CRMP2 loss of function (Inagaki et al., 2001). However, expression of two Pak1 shRNAs affected the segregation of Tau-1 and Map2, indicating its requirement for a neuron to transition from a nonpolarized to a polarized stage. Loss of Pak1 also inhibited outgrowth of all neurites and caused neuronal death, indicating its essential role during neuronal differentiation. These consequences ranged in severity, which likely reflects the differentiation status of individual neurons at the time of dissociation from the hippocampus, the levels of shRNA expression, and the halflife of endogenous Pak1 protein that had been expressed before mRNA degradation. Our findings, together with published literature, suggest that in neurons Pak1 initially acts to promote the specification and rapid outgrowth of an axon. Subsequently, Pak1 is necessary to promote dendrite elaboration where it maintains its postsynaptic function through adulthood by regulating spine morphology and density (Hayashi et al., 2002; Penzes et al., 2003; Zhang et al., 2005). A similar functional switch from axon to dendrites has been reported for the small GTPase Rab8 (Huber et al., 1993, 1995).

Pak1Caax-expressing neurons assumed a star-like morphology as a result of the reduced outgrowth of the future axon and extension of the remaining neurites that contained both Tau-1 and Map2. Interestingly, a similar morphology and distribution of Map2 and Tau-1 was reported after loss of the neuronal microtubule-regulating SAD-A and SAD-B kinases (Kishi et al.,
2005). To date there are no known links between SAD and Pak1 kinase functions, and a clear role for Pak1 as a regulator of microtubules has not been established. The massive accumulation of stabilized, looped microtubules around the cell soma of Pak1 shRNA-expressing neurons may be a direct consequence of its loss or a secondary effect caused by the appearance of extensive lamellipodia. However, the similarity between Pak1 gain of function and SAD loss of function is intriguing and suggests their mutually antagonizing relationship.

Membrane localization of Pak1 can induce neurite outgrowth in PC12 cells regardless of its kinase activity (Daniels et al., 1998). We reveal that the effect of Pak1 on the polarization of hippocampal neurons requires its kinase activity and membrane localization. The fact that PC12 cells do not polarize may account for the observed differences, suggesting that in neurons polarity and outgrowth have a molecular link, part of which may involve the regulated activation of Pak1. It is striking that the highly active Pak1T423E mutant did not alter axonal specification despite a clear induction of actin polymerization, further confirming that polarization requires activation of Pak1 at the membrane. In cortical neurons, Pak1 can promote elaboration of primary dendrites as a major downstream target of Cdc42 and Racl (Hayashi et al., 2002). We did not observe an increase in the number of primary neurites after Pak1 gain of function, although Pak1Caax did induce an increase in dendritic outgrowth. Hayashi et al. (2002) examined neurons that had been electroporated in utero with cytoplasmic Pak1 and subsequently analyzed after dissociation from mouse cortices. These experimental differences may account for our nonoverlapping results. We did, however find that long-term loss of Pak1 severely affected dendritic growth, thus supporting the proposal that Pak1 is required for dendrite formation and function.

A Cdc42 mutant that autonomously cycles between a GTPand GDP-bound state (Cdc42L28) can induce multiple axons downstream of the small GTPase Rap1b (Schwamborn and Puschel, 2004). Additional upstream signals include PI3K and the insulin-like growth factor receptor-1, the loss of which prevent axon formation (Arimura and Kaibuchi, 2005; Wiggin et al., 2005; Sosa et al., 2006). It is therefore likely that Pak1 controls neuronal polarity, at least in part, as a Cdc42 effector. The fact that RacN17 can rescue the Pak1Caax-induced defects in axon specification suggests that it lies downstream rather than upstream of Pak1 during neuronal polarization. DOCK7 was recently identified as the activator of Racl required for the inhibition of stathmin/Op18 and the consequent stabilization of microtubules during the formation of an axon (Watabe-Uchida et al., 2006). Pak1 is a well known inhibitor of stathmin/Op18 and thus a likely regulator in polarizing neurons (Fig. 10) (Wittmann et al., 2003). However, Watabe-Uchida et al. (2006) concluded that Pak1 is not required for the establishment of neuronal polarity, because expression of its autoinhibitory domain (AID) did not rescue the effects of DOCK7 overexpression (Watabe-Uchida et al., 2006). Activation of Pak1 is required before the AID can 

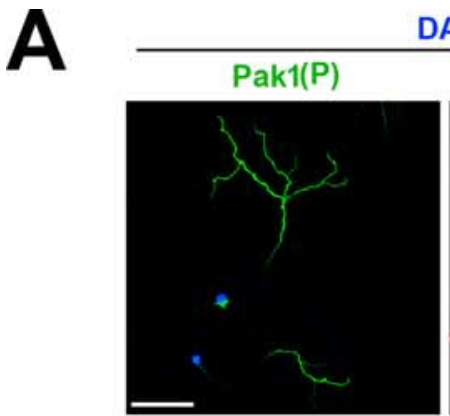

\section{DAPI}
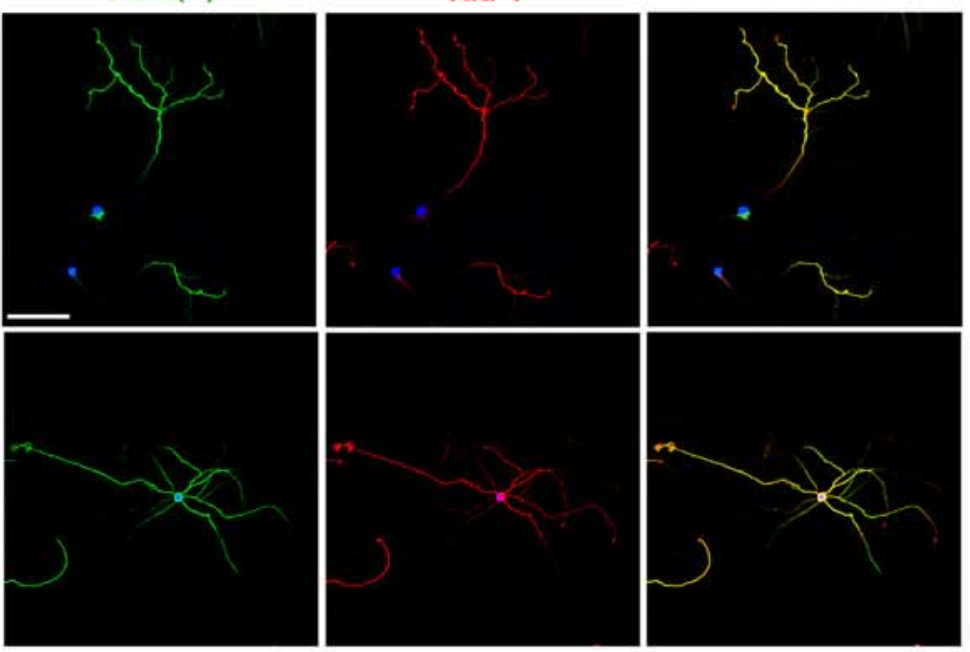

GFP ,Tau-1, DAPI
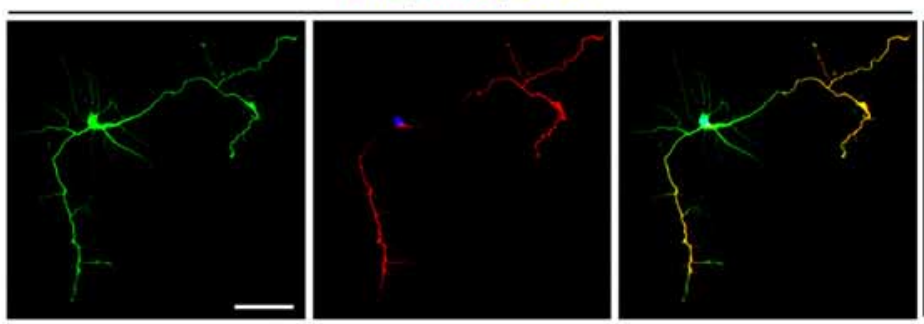

$\frac{x}{\stackrel{\varpi}{0}}$
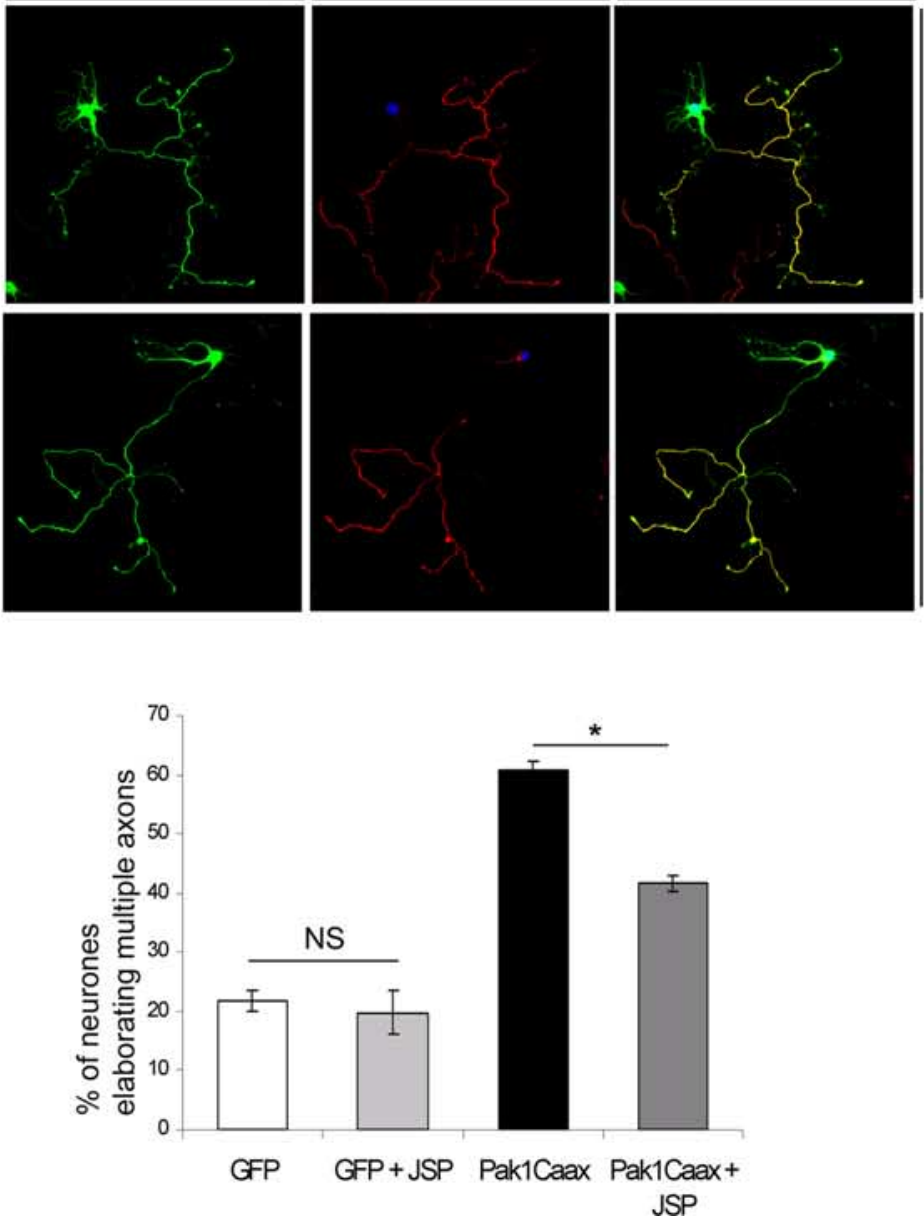

bind, and the Pak1 AID may also associate with Pak2 and Pak3, which can reduce its effectiveness in neurons. Consequently, we used the shRNA approach, ensuring specific targeting of Pak1 mRNA, thus preventing the bulk of its protein synthesis. The fact that two Pak1 shRNA constructs caused similar consequences, whereas a nonspecific shRNA was indistinguishable from an EGFP-expressing control, further supports the effectiveness of our approach (Li et al., 2003). Our data suggest that Pak1 promotes the stage 2 to stage 3 transition in polarizing neurons. Interestingly, the JNK kinases were recently identified as essential regulators of axon specification and neuronal transition from stage 2 to 3 and thus may be neuronal Pak1 effectors (Oliva et al., 2006).

In fibroblast and epithelial cells, localized activation of cofilin can increase the number of free barbed ends and induce polarized F-actin polymerization (Dawe et al., 2003; Ghosh et al., 2004). In rat cortical neurons, cofilin activation has been associated with increased outgrowth of the longest neurite (Kuhn et al., 2000; Meberg and Bamburg, 2000). Interestingly, a constitutively active cofilin mutant was less effective at promoting neurite outgrowth than its wild-type form (Meberg and Bamburg, 2000). Furthermore, phosphorylation and thus inactivation of cofilin was recently shown to accompany VEGFinduced neurite outgrowth in rat cortical neurons (Jin et al., 2006). Together, these findings suggest that the biological consequences of cofilin activity can vary depending on the extracellular signaling factors. They also indicate that regulated cofilin function is important during neuronal differentiation. We propose that Pak1 is responsible for controlling cofilin activity during the transition of a neuron from stage 2 to stage 3 . Overexpression of Pak1Caax in a stage 2 neuron therefore results in uniform and constant inactivation

Figure 8. Pak1 induces axon formation by affecting F-actin organization. $\boldsymbol{A}$, Hippocampal neurons were exposed to low doses $(1 \mu \mathrm{M})$ of $C D$ or control DMSO and maintained for 7 div. CD caused the formation of multiple Tau-1-positive axons, all of which were also enriched with Pak1(P). B, Hippocampal neurons transfected with EGFP or Pak1Caax were exposed to $5 \mathrm{~nm}$ jasplakinolide (JP) or control DMSO after attachment to the substrate and cultured for $7 \mathrm{~d}$. Neurons with multiple Tau-1-positive axons were common in Pak1Caaxexpressing cultures but rarer after treatment with jasplakinolide or in EGFP-expressing controls. Scale bars, $50 \mu \mathrm{m}$. C, Quantification of the effects of jasplakinolide (JSP). Error bars represent SEM. ${ }^{*} p<0.001$ using Student's $t$ test. DAPI, 4',6' -Diamidino-2-phenylindole; NS, not significant. 
A
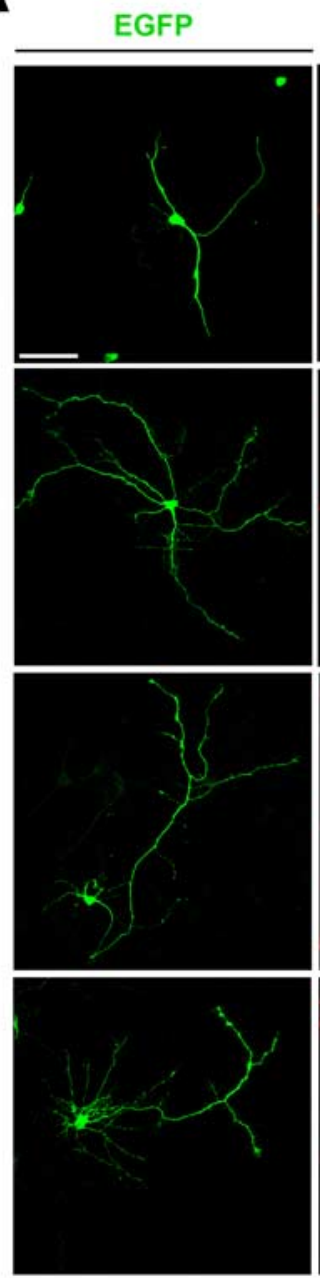

B

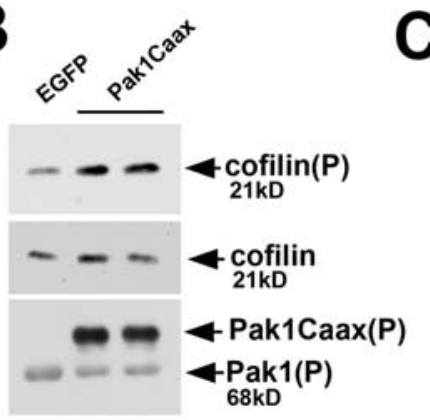

Tau-1
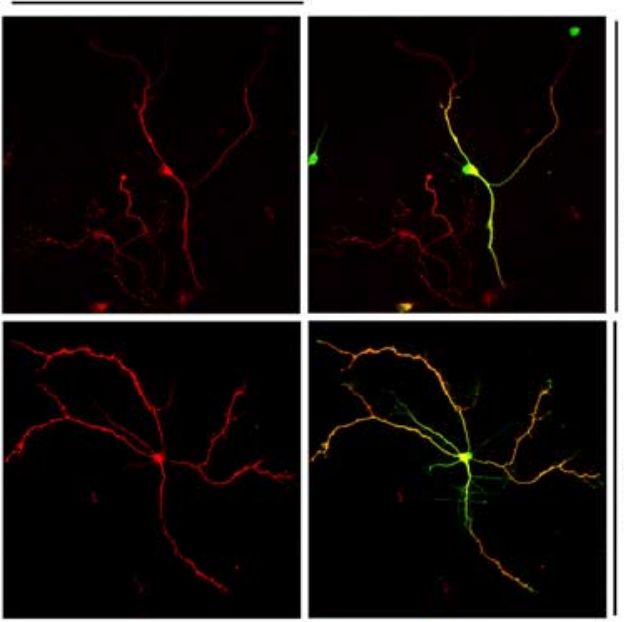

敢
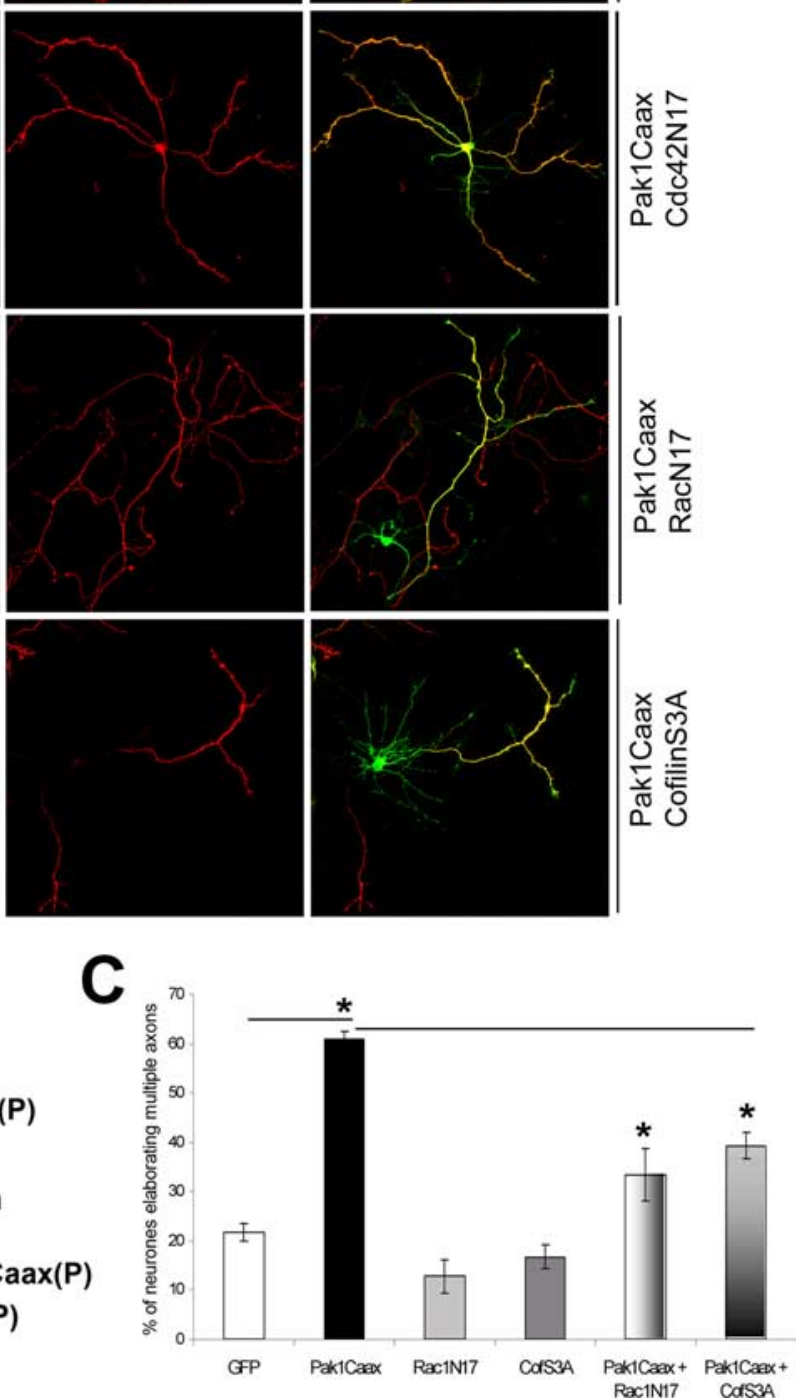

Figure 9. Pak1 induces multiple axons through Rac1 activation and cofilin inhibition. $\boldsymbol{A}$, The consequences of coexpression of RacN17, Cdc42N17, or cofilinS3A mutants with Pak1Caax were examined in hippocampal neurons at 7 div. Both RacN17 and cofilinS3A increased the incidence of neurons elaborating a single axon, which was not observed after Cdc42N17 expression. Scale bar, $50 \mu \mathrm{m}$. B, Cortical neurons expressing EGFP or Pak1Caax were cultured for $7 \mathrm{~d}$ and compared by Western blotting. An approximately threefold increase in S3 phosphorylated cofilin was consistently observed in Pak1Caax-expressing neurons, whereas total cofilin levels remained unchanged. The bottom shows the presence of S199/204 phosphorylated Pak1Caax expression and endogenous Pak1, confirming increased catalytic activity. C, Quantification of RacN17 or cofilinS3A (CofS3A) effects. Scale bar, $50 \mu \mathrm{m}$. Error bars represent SEM. * $p<0.001$ using Student's $t$ test.

of endogenous cofilin. The consequential effects on the F-actin cytoskeleton can be rescued by coexpression of a cofilin mutant that is not subject to Pak1Caax regulation. The restored asymmetry in the F-actin cytoskeleton facilitates axon formation. The effects of Pak1Caax were also rescued by jasplakinolide. In vitro jasplakinolide induces F-actin polymerization; however, in living cells it can also disrupt regulated actin filament elongation by inducing spontaneous aggregates of G-actin (Bubb et al., 2000). Long-term exposure of neurons to low doses of jasplakinolide may protect from the effects of Pak1Caax overexpression by changing the rate of F-actin turnover and its availability for regulation by Pak1.

Together, our data suggest that localized activation of Pak1 is required to generate asymmetric consequences on the F-actin cytoskeleton of a stage 2 neuron. This causes changes in microtubule organization, which are required for the establishment and outgrowth of an axon and dendrites (Fig. 10). We also reveal that Pak1 is essential for neuronal viability.

\section{References}

Arimura N, Kaibuchi K (2005) Key regulators in neuronal polarity. Neuron 48:881-884

Arimura N, Kaibuchi K (2007) Neuronal polarity: from extracellular signals to intracellular mechanisms. Nat Rev Neurosci 8:194-205.

Bokoch GM (2003) Biology of the p21-activated kinases. Annu Rev Biochem 72:743-781.

Bradke F, Dotti CG (1999) The role of local actin instability in axon formation. Science 283:1931-1934.

Bradke F, Dotti CG (2000) Differentiated neurons retain the capacity to generate axons from dendrites. Curr Biol 10:1467-1470.

Bubb MR, Spector I, Beyer BB, Fosen KM (2000) Effects of jasplakinolide on the kinetics of actin polymerization. An explanation for certain in vivo observations. J Biol Chem 275:5163-5170.

Cau J, Hall A (2005) Cdc42 controls the polarity of the actin and microtubule cytoskeletons through two distinct signal transduction pathways. J Cell Sci 118:2579-2587.

Chong C, Tan L, Lim L, Manser E (2001) The mechanism of PAK activation. Autophosphorylation events in both regulatory and kinase domains control activity. J Biol Chem 276:17347-17353.

Daniels RH, Hall PS, Bokoch GM (1998) Membrane targeting of p21-activated kinase 1 (PAK1) induces neurite outgrowth from $\mathrm{PC} 12$ cells. EMBO J 17:754-764.

Da Silva JS, Hasegawa T, Miyagi T, Dotti CG, Abad-Rodriguez J (2005) Asymmetric membrane ganglioside sialidase activity specifies axonal fate. Nat Neurosci 8:606-615.

Dawe HR, Minamide LS, Bamburg JR, Cramer LP (2003) ADF/cofilin controls cell polarity during fibroblast migration. Curr Biol 13:252-257.

DerMardirossian C, Schnelzer A, Bokoch GM (2004) Phosphorylation of RhoGDI by Pak1 mediates dissociation of Rac GTPase. Mol Cell $15: 117-127$

Dharmawardhane S, Brownson D, Lennartz $M$ Bokoch GM (1999) Localization of p21-activated kinase 1 (PAK1) to pseudopodia, membrane ruffles, and phagocytic cups in activated human neutrophils. J Leukoc Biol 66:521-527.

Dotti CG, Sullivan CA, Banker GA (1988) The establishment of polarity by hippocampal neurons in culture. J Neurosci 8:1454-1468.

Edwards DC, Sanders LC, Bokoch GM, Gill GN (1999) Activation of LIM- 
kinase by Pak1 couples Rac/Cdc42 GTPase signalling to actin cytoskeletal dynamics. Nat Cell Biol 1:253-259.

Ghosh M, Song X, Mouneimne G, Sidani M, Lawrence DS, Condeelis JS (2004) Cofilin promotes actin polymerization and defines the direction of cell motility. Science 304:743-746.

Hayashi K, Ohshima T, Mikoshiba K (2002) Pak1 is involved in dendrite initiation as a downstream effector of Racl in cortical neurons. Mol Cell Neurosci 20:579-594.

Hayashi ML, Choi SY, Rao BS, Jung HY, Lee HK, Zhang D, Chattarji S, Kirkwood A, Tonegawa S (2004) Altered cortical synaptic morphology and impaired memory consolidation in forebrain-specific dominant-negative PAK transgenic mice. Neuron 42:773-787.

Holly SP, Blumer KJ (1999) PAK-family kinases regulate cell and actin polarization throughout the cell cycle of Saccharomyces cerevisiae. J Cell Biol 147:845-856.

Huber LA, de Hoop MJ, Dupree P, Zerial M, Simons K, Dotti C (1993) Protein transport to the dendritic plasma membrane of cultured neurons is regulated by rab8p. J Cell Biol 123:47-55.

Huber LA, Dupree P, Dotti CG (1995) A deficiency of the small GTPase rab8 inhibits membrane traffic in developing neurons. Mol Cell Biol 15:918-924.

Inagaki $\mathrm{N}$, Chihara $\mathrm{K}$, Arimura $\mathrm{N}$, Menager $\mathrm{C}$, Kawano Y, Matsuo N, Nishimura T, Amano M, Kaibuchi K (2001) CRMP-2 induces axons in cultured hippocampal neurons. Nat Neurosci 4:781-782.

Jacobson C, Schnapp B, Banker GA (2006) A change in the selective translocation of the Kinesin-1 motor domain marks the initial specification of the axon. Neuron 49:797-804.

Jaffer ZM, Chernoff J (2002) p21-activated kinases: three more join the Pak. Int J Biochem Cell Biol 34:713-717.

Jiang H, Guo W, Liang X, Rao Y (2005) Both the establishment and the maintenance of neuronal polarity require active mechanisms: critical roles of GSK-3beta and its upstream regulators. Cell 120:123-135.

Jin K, Mao XO, Greenberg DA (2006) Vascular endothelial growth factor stimulates neurite outgrowth from cerebral cortical neurons via Rho kinase signaling. J Neurobiol 66:236-242.

Kawauchi T, Chihama K, Nabeshima Y, Hoshino M (2003) The in vivo roles of STEF/Tiam1, Rac1 and JNK in cortical neuronal migration. EMBO J 22:4190-4201.

Kawauchi T, Chihama K, Nabeshima Y, Hoshino M (2006) Cdk5 phosphorylates and stabilizes p27kip1 contributing to actin organization and cortical neuronal migration. Nat Cell Biol 8:17-26.

King CC, Gardiner EM, Zenke FT, Bohl BP, Newton AC, Hemmings BA, Bokoch GM (2000) p21-activated kinase (PAK1) is phosphorylated and activated by 3-phosphoinositide-dependent kinase-1 (PDK1). J Biol Chem 275:41201-41209.

Kishi M, Pan YA, Crump JG, Sanes JR (2005) Mammalian SAD kinases are required for neuronal polarization. Science 307:929-932.

Kuhn TB, Meberg PJ, Brown MD, Bernstein BW, Minamide LS, Jensen JR, Okada K, Soda EA, Bamburg JR (2000) Regulating actin dynamics in neuronal growth cones by ADF/cofilin and rho family GTPases. J Neurobiol 44:126-144.

Lee S, Rivero F, Park KC, Huang E, Funamoto S, Firtel RA (2004) Dictyostelium PAKc is required for proper chemotaxis. Mol Biol Cell 15:5456-5469.

Lei M, Lu W, Meng W, Parrini MC, Eck MJ, Mayer BJ, Harrison SC (2000) Structure of PAK1 in an autoinhibited conformation reveals a multistage activation switch. Cell 102:387-397.

Li Z, Hannigan M, Mo Z, Liu B, Lu W, Wu Y, Smrcka AV, Wu G, Li L, Liu M, Huang CK, Wu D (2003) Directional sensing requires G beta gamma- mediated PAK1 and PIX alpha-dependent activation of Cdc42. Cell 114:215-227.

Loo TH, Ng YW, Lim L, Manser E (2004) GIT1 activates p21-activated kinase through a mechanism independent of p21 binding. Mol Cell Biol 24:3849-3859.

Manser E, Loo TH, Koh CG, Zhao ZS, Chen XQ, Tan L, Tan I, Leung T, Lim L (1998) PAK kinases are directly coupled to the PIX family of nucleotide exchange factors. Mol Cell 1:183-192.

Meberg PJ, Bamburg JR (2000) Increase in neurite outgrowth mediated by overexpression of actin depolymerizing factor. J Neurosci 20:2459-2469.

Nichols CB, Fraser JA, Heitman J (2004) PAK kinases Ste20 and Pak1 govern cell polarity at different stages of mating in Cryptococcus neoformans. Mol Biol Cell 15:4476-4489.

Nikolic M, Chou MM, Lu W, Mayer BJ, Tsai LH (1998) The p35/Cdk5 kinase is a neuron-specific Rac effector that inhibits Pak1 activity. Nature 395:194-198.

Noctor SC, Martinez-Cerdeno V, Ivic L, Kriegstein AR (2004) Cortical neurons arise in symmetric and asymmetric division zones and migrate through specific phases. Nat Neurosci 7:136-144.

Obermeier A, Ahmed S, Manser E, Yen SC, Hall C, Lim L (1998) PAK promotes morphological changes by acting upstream of Rac. EMBO J 17:4328-4339.

Oliva Jr AA, Atkins CM, Copenagle L, Banker GA (2006) Activated c-Jun $\mathrm{N}$-terminal kinase is required for axon formation. J Neurosci 26:9462-9470.

Ottilie S, Miller PJ, Johnson DI, Creasy CL, Sells MA, Bagrodia S, Forsburg SL, Chernoff J (1995) Fission yeast pak1 + encodes a protein kinase that interacts with Cdc42p and is involved in the control of cell polarity and mating. EMBO J 14:5908-5919.

Penzes P, Beeser A, Chernoff J, Schiller MR, Eipper BA, Mains RE, Huganir RL (2003) Rapid induction of dendritic spine morphogenesis by transsynaptic ephrinB-EphB receptor activation of the Rho-GEF kalirin. Neuron 37:263-274.

Rashid T, Banerjee M, Nikolic M (2001) Phosphorylation of Pak1 by the p35/Cdk5 kinase affects neuronal morphology. J Biol Chem 276:49043-49052. 
Sarmiere PD, Bamburg JR (2004) Regulation of the neuronal actin cytoskeleton by ADF/cofilin. J Neurobiol 58:103-117.

Schwamborn JC, Puschel AW (2004) The sequential activity of the GTPases Rap1B and Cdc42 determines neuronal polarity. Nat Neurosci 7:923-929.

Sells MA, Boyd JT, Chernoff J (1999) p21-activated kinase 1 (Pak1) regulates cell motility in mammalian fibroblasts. J Cell Biol 145:837-849.

Sells MA, Pfaff A, Chernoff J (2000) Temporal and spatial distribution of activated Pak1 in fibroblasts. J Cell Biol 151:1449-1458.

Solecki DJ, Govek EE, Tomoda T, Hatten ME (2006) Neuronal polarity in CNS development. Genes Dev 20:2639-2647.

Sosa L, Dupraz S, Laurino L, Bollati F, Bisbal M, Caceres A, Pfenninger KH, Quiroga S (2006) IGF-1 receptor is essential for the establishment of hippocampal neuronal polarity. Nat Neurosci 9:993-995.

ten Klooster JP, Jaffer ZM, Chernoff J, Hordijk PL (2006) Targeting and activation of Racl are mediated by the exchange factor beta-Pix. J Cell Biol 172:759-769.

Trivedi N, Marsh P, Goold RG, Wood-Kaczmar A, Gordon-Weeks PR (2005) Glycogen synthase kinase-3beta phosphorylation of MAP1B at Ser1260 and Thr1265 is spatially restricted to growing axons. J Cell Sci 118:993-1005.
Tsai LH, Gleeson JG (2005) Nucleokinesis in neuronal migration. Neuron 46:383-388.

Watabe-Uchida M, John KA, Janas JA, Newey SE, Van Aelst L (2006) The Rac activator DOCK7 regulates neuronal polarity through local phosphorylation of stathmin/Op18. Neuron 51:727-739.

Wiggin GR, Fawcett JP, Pawson T (2005) Polarity proteins in axon specification and synaptogenesis. Dev Cell 8:803-816.

Wittmann T, Bokoch GM, Waterman-Storer CM (2003) Regulation of leading edge microtubule and actin dynamics downstream of Rac1. J Cell Biol 161:845-851.

Zhang H, Webb DJ, Asmussen H, Niu S, Horwitz AF (2005) A GIT1/PIX/ Rac/PAK signaling module regulates spine morphogenesis and synapse formation through MLC. J Neurosci 25:3379-3388.

Zhao ZS, Lim JP, Ng YW, Lim L, Manser E (2005) The GIT-associated kinase PAK targets to the centrosome and regulates Aurora-A. Mol Cell 20:237-249.

Zhong JL, Banerjee MD, Nikolic M (2003) Pak1 and its T212 phosphorylated form accumulate in neurones and epithelial cells of the developing rodent. Dev Dyn 228:121-127. 